\title{
Classification of Diffuse Lung Disease Patterns on High-Resolution Computed Tomography by a Bag of Words Approach
}

\author{
Rui $\mathrm{Xu}^{1}$, Yasushi Hirano ${ }^{1}$, Rie Tachibana ${ }^{2}$, and Shoji Kido ${ }^{1}$ \\ 1 Applied Medical Engineering Science, Graduate School of Medicine, \\ Yamaguchi University, Ube, Japan \\ \{xurui, yhirano,kido.ai\}@yamaguchi-u.ac.jp \\ 2 Information Science and Technology Dept., \\ Oshima National College of Maritime Technology, Oshima-Gun, Japan \\ tatibana@oshima-k.ac.jp
}

\begin{abstract}
Visual inspection of diffuse lung disease (DLD) patterns on high-resolution computed tomography (HRCT) is difficult because of their high complexity. We proposed a bag of words based method on the classification of these textural patters in order to improve the detection and diagnosis of DLD for radiologists. Six kinds of typical pulmonary patterns were considered in this work. They were consolidation, ground-glass opacity, honeycombing, emphysema, nodular and normal tissue. Because they were characterized by both CT values and shapes, we proposed a set of statistical measure based local features calculated from both CT values and the eigen-values of Hessian matrices. The proposed method could achieve the recognition rate of $95.85 \%$, which was higher comparing with one global feature based method and two other CT values based bag of words methods.
\end{abstract}

\section{Introduction}

Diffuse lung disease (DLD) refers to a group of lung disorders which spread out in large areas. In the detection and diagnosis of DLD, high-resolution computed tomography (HRCT) has played important roles in recent years [12. Thin slice $\mathrm{CT}$ is able to give detailed appearances on pulmonary patterns characterized for specific abnormal findings which point toward a specific diagnosis and treatment. In current clinical protocols, the objective identification of such patterns has not being established, and visual inspection is carried on, according to the experiences of radiologists. Not only subjective differences lead to inevitable misjudgements, but also huge amount of images makes a big burden on radiologists. Therefore, a quantitative computer-aided diagnosis (CAD) tool is required to give the second opinion to facilitate the detection and diagnosis of DLD.

There were researches on computer-aided analysis of DLD patterns in the past ten years [9]. From the viewpoints of computer vision, it can be generalized as the problem of texture analysis on a certain $2 \mathrm{D}$ or $3 \mathrm{D}$ region of interest (ROI). Some classical textural feature analysis methods calculated on 2D ROIs

G. Fichtinger, A. Martel, and T. Peters (Eds.): MICCAI 2011, Part III, LNCS 6893, pp. 183-190, 2011. (C) Springer-Verlag Berlin Heidelberg 2011 

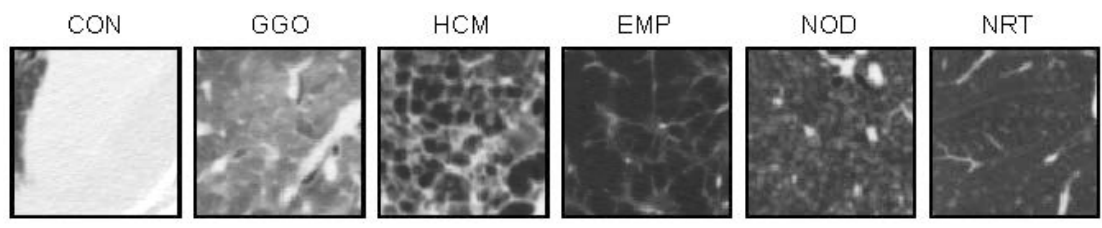

Fig. 1. Examples of six kinds of pulmonary patterns in HRCT

can be used, including the direct analysis on gray value intensities [2, features calculated on histogram statistics, and features extraction based on filter-banks, co-occurrence matrices or run-length parameters [1] [5] [13. Features may also be designed for the specific task on hands [4]. Different from the above-mentioned methods which analyzed on 2D ROIs, a method based on features calculated on $3 \mathrm{D}$ ROIs was proposed in 10, which reported that 3D ROIs based features were more sensitive and specific. All of these textural analysis methods made use of global features.

As the development of computer vision techniques, there have been more and more researches which reported that techniques based on local features could lead to more accurate results on recognition tasks [11. Local features are less sensitive to the changing of illuminations and positions, so they usually could be a more robust way to represent images of underlying objects. An efficient approach to use local features is called bag of words (keypoints) [6]. It was firstly proposed in the area of statistical natural language analysis [3], then introduced into the area of computer vision in [6] for many applications.

Recently, local features have been considered on the analysis of pulmonary patterns on HRCT. Local binary patterns (LBP) combined with $k$ nearest neighbor $(k-\mathrm{NN})$ was adopted to distinguish three kinds of pulmonary patterns (normal tissues, centrilobular emphysema and paraseptal emphysema) for chronic obstructive pulmonary disease (COPD) in [16]. A bag of words approach (originally called texton-based method) was also tried in the same problem in [15]. According to our knowledge, there are no works to apply local features on DLD patterns analysis. Compared to [15, the main differences are listed as follows:

- our aim is to classify pulmonary patterns for DLD rather than COPD.

- more categories of pulmonary patterns.

- local features extracted from 3D ROIs instead of 2D ROIs.

- compact and efficient statistical measure based local features considering both shapes and CT values.

Fig. 1 I gives the examples of six types of typical pulmonary patterns in HRCT for DLD. They are consolidation (CON), ground-glass opacity (GGO), honeycombing (HCM), emphysema (EMP), nodular (NOD) and normal tissue (NRT). The aim of our work is to find an efficient computer-aided analysis method to distinguish them in order to improve the detection and diagnosis of DLD on HRCT. 


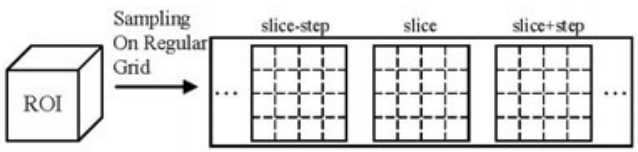

(a). Local features calculated at regular sampling grids in each ROI.

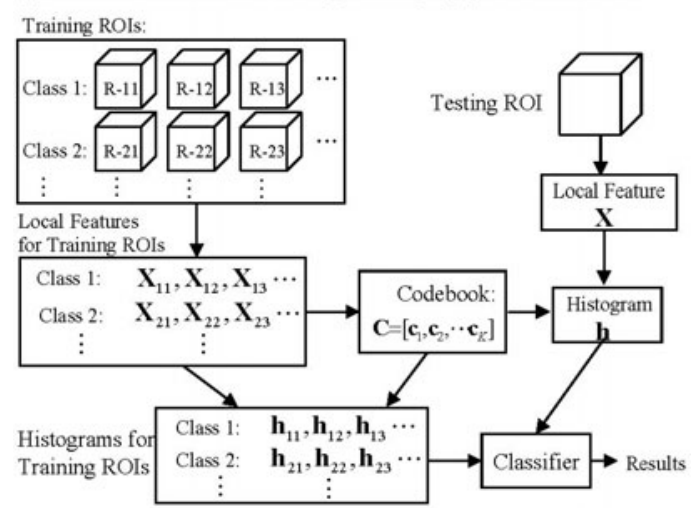

(b). Diagram of training and testing Procedures in the bag of words approach.

Fig. 2. The framework of the bag of words approach

\section{Method}

\subsection{Bag of Words Approach}

Fig 2 describes the framework of bag of words approach, which is composed by three main steps, local feature calculation, code-book construction and classification. Local features can be calculated at the regularly sampling grids or at the pre-determined key-point positions. According to literature [11, there seems to be no evidence to show which one is better. In this work, we prefer to adopt the way of regularly sampling grids, since it is easier and faster. Here, the ROI size and the grid step is set to be $32 \times 32 \times 16$ and $4 \times 4 \times 4$ respectively, so we can get $9 \times 9 \times 5=405$ sampling points on one ROI. For each sampling point, one local feature is calculated in a patch whose center is located on it. Therefore, 405 local features can be calculated on each ROI. The patch size is the parameter determined by experiments.

The main idea of bag of words approach is to represent the ROIs (or images) by a histogram whose bins are the elements of a code-book trained from local features. Such a code-book can be seen as an intermediate layer to interpret images. Since local features are calculated from limited local regions, each of them only reflects information of partial objects. Although local features are huge, they are usually clustered into limited number of centers in high-dimensional feature spaces. Just as words are basic elements of sentences, such centers of clusters can also be considered as the basic elements which compose the underlying objects. This is the reason why it is called bag of words approach. The unit of these elements is called a code-book or dictionary. Construction of such a 
dictionary can be generalized as a vector quantization problem. K-means method is adopted to resolve this problem. The number of clusters in K-means $(K)$ is another parameter determined by experiments.

In the classification step, all of the local features of one ROI are assigned to a label which indicates the nearest elements (or words) in the code-book. Given each elements as the bins, a histogram can be calculated to refer to the statistical information of the ROI. Since the same type of ROIs gives similar histograms, such histograms can be treated as input vectors for the classification.

In our opinion, bag of words approach is a common framework. According to different recognition tasks, the method of local feature extraction and classifier should be adjusted in order to make it achieve better results. We will dwell on these two aspects in the following two subsections.

\subsection{Local Features}

CT values of a squared patch was treated as local features for each 2D ROIs in 15. It was reported that such local features achieved good results for the recognition of normal tissues and two kinds of emphysema patterns. We tried this method, but the results were not satisfied. Therefore, we reconsidered about our problem and designed a new kind of local feature extraction method suitable for our task.

The categories of pulmonary patterns in our work are more than [15. Some patterns, such as NRT, CON and EMP, are mainly characterized by CT values. However, shape information should be taken into consideration in order to distinguish the patterns, such as HCM and NOD. Since shape information can not be shown from 2D slices, 3D ROIs are adopted in this work. An usual way to describe shape information is to use the eigen-values of Hessian matrices calculated on 3D ROIs. These eigen-values can be arranged to form three cubes according to the arrangement of the original voxels. In order to consider the information of both $\mathrm{CT}$ values and shapes, local features are calculated from both the three eigen-value cubes and the original ROI. Four kinds of statistical measures, mean, variance, skewness and kurtosis are calculated from a cube-patch centered at the regular sampling grids. Therefore, one local features consists of 16 statistical measures. The size of cube-patch is the parameter for this local feature extraction method and its optimal value is determined by experiments. It should be noted that no matter how it changes, the compactness of such local features does not change. Additionally, these statistical measures are invariant to translation and rotation. Here, the Hessian matrix was only calculated on the pixel level.

\subsection{Classifier}

SVM was adopted as the classifier in this work. We used a version called LIBSVM [17]. For a generalized recognition problem, a common choice of the kernel for SVM is a Gaussian kernel. For the bag of words problem, it was reported that some other kernels was superior to Gaussian kernel if input vectors were 
histograms [14]. We tried other three kinds of kernels. The kernel definitions are given by Eq[1]

$$
\left\{\begin{array}{l}
G\left(\mathbf{h}, \mathbf{h}^{\prime}\right)=\exp \left(-\gamma\left\|\mathbf{h}-\mathbf{h}^{\prime}\right\|^{2}\right) \\
K_{1}\left(\mathbf{h}, \mathbf{h}^{\prime}\right)=\sum_{i=1}^{N} \min \left(h_{i}, h_{i}^{\prime}\right) \\
K_{2}\left(\mathbf{h}, \mathbf{h}^{\prime}\right)=\sum_{i=1}^{N} \sqrt{h_{i} h_{i}^{\prime}} \\
K_{3}\left(\mathbf{h}, \mathbf{h}^{\prime}\right)=\exp \left(-\alpha \sum_{i=1}^{N} \frac{\left(h_{i}-h_{i}^{\prime}\right)^{2}}{h_{i}+h_{i}^{\prime}}\right)
\end{array}\right.
$$

where $\mathbf{h}=\left[h_{1}, \ldots, h_{N}\right]^{T}$ is the $N$-bin histogram. $G\left(\mathbf{h}, \mathbf{h}^{\prime}\right)$ is the Gaussian kernel, $K_{1}\left(\mathbf{h}, \mathbf{h}^{\prime}\right)$ is the histogram intersection kernel, $K_{2}\left(\mathbf{h}, \mathbf{h}^{\prime}\right)$ is the Bhattacharyya kernel, $K_{3}\left(\mathbf{h}, \mathbf{h}^{\prime}\right)$ is the $\chi^{2}$-kernel. $\gamma$ is the parameter for the gaussian kernel and $\alpha$ is the parameter for the $\chi^{2}$-kernel. Additionally, a soft margin parameter $C$ is considered for all the four kernels. These parameters will be optimized in experiments.

\section{Experiments and Results}

\subsection{Data}

We obtained 117 scans for different subjects from Tokushima University Hospital, Tokushima, Japan. All of them were scanned from 16-row multi-slice CT (Aquilion, Toshiba Co.), when edge-enhanced filtering was not applied. The resolution was $512 \times 512$ with the pixel size of $0.6 \mathrm{~mm}$ on each slice, and the slice-thickness was $1 \mathrm{~mm}$. The regions of the six types of patterns were marked by three experienced radiologists according to the following procedure. Firstly, one radiologist was asked to review all scans. From each scan, maximum of three slices were selected where typical patters dominantly spread. Then together with the other two radiologists, the six kinds of patters were marked on the selected slices separately. Finally, the common regions marked by all radiologists were considered as where typical patterns were located. The ROIs were constructed according to these determined regions. The centers of ROIs were randomly selected from them while considering non-overlap on ROIs. At last, 3009 3D-ROIs were determined.

\subsection{Results and Discussion}

There were mainly three kinds of parameters which should be adjusted by experiments in our proposed method. They were the size of cube-patches, the number of clusters in K-means, and the parameters related to SVM classifiers (including kernel types, the soft margin parameter, and parameters for each kernel). These parameters were determined by a 20 -fold cross-validation test. First, by setting number of clusters to be a certain number (100 was used) and the kernel type of SVM to be the Gaussian kernel, we exhausted the possible values on the size of cube-patches, soft margin parameter and $\gamma$ of the gaussian kernel to train classifiers. The size of cube-patches was optimized by testing on the training data of 20-fold cross-validation tests. Second, by using the optimized cube-patch 


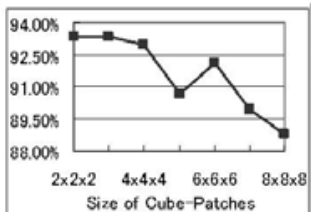

(a)

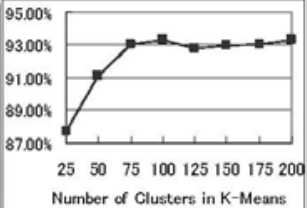

(b)

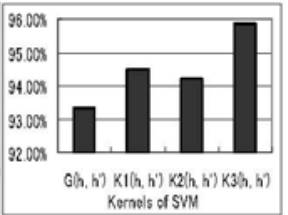

(c)

Fig. 3. The choices of parameters in our method

size and setting the classifier to be Gaussian kernel based SVM again, we tried all the possible values on the number of clusters and parameters related to the classifier to optimize the number of clusters in the same way. Finally, the parameters related to SVM were optimized, when the cube-patch size and the number of clusters were set to be the optimized values.

For the size of the cube-patches, we tried the size from $2 \times 2 \times 2$ to $8 \times 8 \times 8$. Fig. 3 (a) gives the results when different patch sizes were used. Considering that more voxels could give more stable statistical measures, the size of $3 \times 3 \times 3$ was determined in this work. On the choice of the number of clusters in K-Means, we tried the number from 25 to 200 (Fig. 3 (b)) and chose 100 as the optimized value. According to the experimental results, shown by Fig. 3. (c), the $\chi^{2}$-kernel $\left(K_{3}\left(\mathbf{h}, \mathbf{h}^{\prime}\right)\right)$ gave a little better results than the other three kernels. We chose the $\chi^{2}$-kernel as the optimal kernel for the SVM classifier.

We also compared our method with one global feature based method (Glo3D) and two bag of words methods (CTV-2D and CTV-3D) which only made use of the CT values. In order to fairly compare them with our method, all their parameters were optimized by the same way used for our method. A brief introduction about them is listed as follows:

- CT Values on 2D Patches (CTV-2D). We implemented a similar version of the method proposed in 15. The ROI was a 2D slice whose center was the same as the 3D-ROI used in this work. The CT values in 2D squared-patches were arranged to be a vector as local features. According to [15], SVM with Gaussian kernels was adopted as the classifier. The parameters are the size of squared-patches, the number of clusters, the soft margin parameter and the $\gamma$ of Gaussian kernel.

- CT Values on 3D Patches (CTV-3D). This method was similar to CTV-2D. The difference was that a 3D ROI was used instead of a 2D ROI. CT values based local features and Gaussian kernel based SVM were used. The parameters are the size of cube-patches, the number of clusters, the soft margin parameter and the $\gamma$ of Gaussian kernel.

- Global Features on 3D Patches (Glo-3D). This was not a bag of words approach. Global features calculated directly from a 3D ROI consisted of two kinds of common used textural features, measures on gray-level cooccurrence matrices (GLCM) [7] and measures on gray-level run-length matrices (GLRLM) [8]. The two kinds of measures were concatenated to be a 
Table 1. Recognition results in experiments

(a) Comparison of four methods

\begin{tabular}{|c|c|}
\hline Methods & Accuracy \\
\hline CTV-2D & $86.71 \%$ \\
\hline CTV-3D & $91.12 \%$ \\
\hline Glo-3D & $91.96 \%$ \\
\hline Ours & $95.85 \%$ \\
\hline
\end{tabular}

(b) Confusion table of our method

\begin{tabular}{|c|c|c|c|c|c|c|c|}
\hline & \multicolumn{6}{|c|}{ Estimated Labels } & \\
\hline True Labels & $\mathrm{CON}$ & GGO & $\mathrm{HCM}$ & EMF & $\mathrm{NOD}$ & NRT & Accuracy \\
\hline $\mathrm{CON}$ & 122 & 1 & 1 & 0 & 0 & 0 & $98.39 \%$ \\
\hline GGO & 1 & 494 & 2 & 1 & 8 & 5 & $96.67 \%$ \\
\hline HCM & 0 & 2 & 520 & 3 & 1 & 0 & $98.86 \%$ \\
\hline EMP & 0 & 3 & 5 & 710 & 6 & 13 & $96.34 \%$ \\
\hline NOD & 1 & $\overline{9}$ & $\overline{0}$ & 11 & 313 & 26 & $86.94 \%$ \\
\hline NRT & 0 & 5 & 0 & 2 & 19 & 725 & $96.54 \%$ \\
\hline
\end{tabular}

feature vector for recognition. SVM with Gaussian kernels was adopted as the classifier. The parameters are the soft margin parameter and the $\gamma$ of Gaussian kernel.

Using the parameters optimized in training, we compared the four methods by testing on the testing data in the 20 -fold cross-validation tests. Table 1(a) gives the comparison results. It can be seen that our methods gave higher recognition accuracy than the other three methods. It should be noted that Glo-3D gave better results than the two bag of words based methods, CTV-2D and CTV-3D. This is because that the only consideration of CT values in local features can not distinguish these patterns and affects the performances of bag of words approaches. Table 1(b) gives the confusion table for each kinds of patterns for our method.

\section{Conclusion}

We proposed a bag of word approach to automatically classify six kinds of pulmonary patterns on HRCT for DLD. Some patterns, such as CON, NRT and EMP, were mainly characterized by CT values; while for other patterns, such as NOD and HCM, both CT values and shape information should be considered in order to classify them successfully. According to such a consideration, we proposed a new kind of local features calculated from both the original CT values and eigen-values of Hessian matrices for our bag of words approach. Experimental results showed that this method was superior to other two kinds of bag of words approaches which only depended on CT values and one global feature based method. We will try some other local features, or combine both local and global features in our future research.

Acknowledgement. This research was supported by a grand-in-aid for scientific research from the Ministry of Education, Culture, Sports, Science, and Technology, Japan, under the grand No. 21103008.

\section{References}

1. Uppaluri, R., Heitmman, E.A., Sonka, M., Hartley, P.G., Hunninghake, G.W., Mclennan, G.: Computer Recognition of Regional Lung Disease Patterns. American Journal of Respiratory and Critical Care Medicine 160(2), 648-654 (1999) 
2. Kauczor, H., Heitmann, K., et al.: Automatic Detection and Quantification of Ground-glass opacities on High-resolution CT Using multiple Neural Networks: Comparison with a Density Mask. American Journal of Roentgenology 175(5), 1329-1334 (2000)

3. Manning, C.D., Schfutze, H.: Foundation of Statistical Natural Language Processing. The MIT Press, Cambridge (2001)

4. Uchiyama, Y., Katsuragawa, S., Abe, H., Shiraishi, J., Li, F., Li, Q., Zhang, C., Suzuki, K., Doi, K.: Quantitative Computerized Analysis of Diffuse Lung Disease in High-resolution Computed Tomography. Medical Physics 30(9), 2440-2454 (2003)

5. Sluimer, I.C., Waes, P.F.V., Viergever, M.A., Ginneken, B.V.: Computer-aided Diagnosis in High-resolution CT of the Lungs. Medical Physics 30(12), 3081-3090 (2003)

6. Csurka, G., Dance, C.R., Fan, L., Bray, C.: Visual Categorization with Bags of Keypoints. In: Proc. of ECCV Workshop on Statistical Learning in Computer Vision, pp. 1-22 (2004)

7. Kurani, A.S., Xu, D., Furst, J.D., Raicu, D.S.: Co-occurrence matrices for volumetric data. In: 7th IASTED International Conference on Computer Graphics and Imaging, Hawai (2004)

8. Xu, D., Kurani, A.S., Furst, J.D., Raicu, D.S.: Run-length encoding for volumetric texture. In: The 4th IASTED International Conference on Visualization, Imaging, and Image Processing (2004)

9. Sluimer, I., Schilham, A., Prokop, M., Ginneken, B.V.: Computer Analysis of Computed Tomography Scans of the Lung: A Survey. IEEE Transactions on Medical Imaging 25(4), 385-405 (2006)

10. Xu, Y., Sonka, M., McLennan, G., Guo, J.F., Hoffman, E.A.: MDCT-based 3-D Texture Classification of Emphysema and Early Smoking Related Lung Pathologies. IEEE Transactions on Medical Imaging 25(4), 464-475 (2006)

11. Tuytelaars, T., Mikolaczyk, K.: Local Invarriant Feature Detectors: A Survey. Foundations and Trands in Computer Graphics and Vision 3(3), 177-280 (2007)

12. Webb, W., Müller, N.L., Naidich, D.: High Resolution CT of the Lung, 4th edn. Lippincott Williams \& Wilkins, Baltimore (2008)

13. Park, S.O., Seo, J.B., Kim, N., Park, S.H., Lee, Y.K., Park, B.W., Sung, Y.S., Lee, Y., Kang, J., Lee, J., Kang, S.H.: Feasibility of Automated Quantification of Regional Disease Patterns Depicted on High-resolution Computed Tomography in Patients with Various Diffuse Lung Diseases. Korean Journal of Radiology 10(5), 455-463 (2009)

14. Lampert, C.H.: Kernel Methods in Computer Vision. Foundations and TrendsR in Computer Graphics and Vision 4(3), 193-285 (2009)

15. Gangeh, M.J., Sørensen, L., Shaker, S.B., Kamel, M.S., Bruijne, M.D., Loog, M.: A Texton-Based Approach for the Classification of Lung Parenchyma in CT Images. In: Jiang, T., Navab, N., Pluim, J.P.W., Viergever, M.A. (eds.) MICCAI 2010. LNCS, vol. 6363, pp. 595-602. Springer, Heidelberg (2010)

16. Sørensen, L., Shaker, S.B., de Bruijne, M.: Quantitative analysis of pulmonary emphysema using local binary patterns. IEEE Transaction on Medical Imaging 29(2), 559-569 (2010)

17. Chang, C.C., Lin, C.J.: LIBSVM : a library for support vector machines, http://www.csie.ntu.edu.tw/ cjlin/libsvm 